\title{
PERTURBATIONS OF THE UNILATERAL SHIFT AND TRANSITIVE OPERATOR ALGEBRAS
}

\author{
MOHAMAD A. ANSARI \\ (Communicated by John B. Conway)
}

\begin{abstract}
An operator $T$ on a Hilbert space $\mathscr{H}$ is said to have the transitive algebra property if $\mathscr{L}(\mathscr{H})$ is the only transitive operator algebra which contains $T$. It was shown by Arveson that the unilateral shift has this property. It is the purpose of the present paper to show that perturbations of the unilateral shift by a large class of finite rank operators have the transitive algebra property. Our results are partial solutions of the well-known transitive algebra problem.
\end{abstract}

1. Introduction. An algebra of operators on a separable, infinite-dimensional, and complex Hilbert space is transitive if it has only the trivial invariant subspaces. The transitive algebra problem is the question: Must every transitive operator algebra be strongly dense in the algebra of all operators? This question is still open. However, many authors have obtained partial solutions of this problem by adding some additional hypothesis. For a survey of results see $[\mathbf{1}, \mathbf{3}, \mathbf{4}, \mathbf{5}, \mathbf{6}$, and 7, Chapter 8].

In the present paper, we will introduce the concept of the strong transitive algebra property (strong-TAP) and we will show that a large class of finite rank operators has the strong-TAP. Indeed, we will obtain partial solutions of the transitive algebra problem by showing: Transitive operator algebras containing certain finite rank perturbations of the unilateral shift are strongly dense in the algebra of all operators. We will conclude by listing open questions which arise from our work.

2. Preliminaries. Throughout, $\mathscr{H}$ denotes a complex Hilbert space with a fixed orthonormal basis $\left\{e_{j}\right\}_{j=1}^{\infty}$ and $S \in \mathscr{L}(\mathscr{H})$ denotes the shift operator $\left(S e_{j}=e_{j+1}\right)$.

DEFINITION 2.1. An operator $T \in \mathscr{L}(\mathscr{H})$ is said to have the transitive algebra property (TAP) if whenever $\mathscr{U}$ is a transitive operator algebra such that $T \in \mathscr{U}, \mathscr{U}$ is strongly dense in $\mathscr{L}(\mathscr{H})$.

It was shown by Arveson [4] that the shift operator has the TAP.

Definition 2.2. An operator $T \in \mathscr{L}(\mathscr{H})$ is said to have the strong transitive algebra property (strong-TAP) if both operators $T$ and $S+T$ have the TAP.

Received by the editors May 22, 1986 and, in revised form, August 11, 1986. This paper was presented at the AMS annual meeting January, 1987 (San Antonio).

1980 Mathematics Subject Classification (1985 Revision). Primary 47C05; Secondary 46Cxx.

This research was supported in part by a grant from Pennsylvania State University.

1987 American Mathematical Society $0002-9939 / 87 \$ 1.00+\$ .25$ per page 
If $T \in \mathscr{L}(\mathscr{H})$ and $\mathscr{M}$ is a subspace (closed linear manifold) of $\mathscr{H}$, we let $\vee T \mathscr{M}$ denote the subspace of $\mathscr{H}$ spanned by the (possibly nonclosed) linear manifold $T \mathscr{M}$.

Finite rank operators have the TAP [7, p. 147]; we will show that certain classes of finite rank operators have the strong-TAP. Throughout, we will use the following result.

THEOREM 2.1 [1]. Let $\mathscr{H}$ and $\mathscr{K}$ be two Hilbert spaces, and let $\mathscr{A}$ be a subset of $\mathscr{L}(\mathscr{H} \oplus \mathscr{K})$ consisting of operators of the form $\left[\begin{array}{cc}A & B \\ 0 & 0\end{array}\right]$ with the following condition:

$$
\bigvee\left\{A \mathscr{H}+B \mathscr{K}:\left[\begin{array}{cc}
A & B \\
0 & 0
\end{array}\right] \in \mathscr{A}\right\}=\mathscr{H} .
$$

If $T$ is an operator in $\mathscr{L}(\mathscr{H} \oplus \mathscr{K})$, if $\mathscr{H} \in \mathscr{L}$ at $(T)$, and if $T \mid \mathscr{H}$ has the TAP, then $\mathscr{L}(\mathscr{H} \oplus \mathscr{K})$ is the only weakly closed transitive operator algebra which contains $\mathscr{A} \cup\{T\}$.

\section{Main results.}

Definition 3.1. By an almost upper triangular matrix we mean a matrix $U=\left(u_{i j}\right)$ of the form

$$
\left(\begin{array}{ccccc}
* & \cdots & & & * \\
u_{21} & \ddots & & & \vdots \\
0 & u_{32} & & & \\
\vdots & & & & \\
0 & \cdots & 0 & u_{n, n-1} & *
\end{array}\right)
$$

with $u_{i+1, i} \neq-1$ for each $i$.

For each $i$, we let $\mathscr{M}_{i}$ denote the subspace of $\mathscr{H}$ which is spanned by the basis vectors $\left\{e_{j}: j \geqslant i+1\right\}$.

THEOREM 3.1. If $U$ is an almost upper triangular $n \times n(n \geqslant 1)$ matrix, then the operator $U \oplus 0 \in \mathscr{L}(\mathscr{H})$ has the strong transitive algebra property.

Proof. Let $\mathscr{U}$ be a transitive operator algebra which contains the operator $T=S+U \oplus 0$. Observe that we have $\mathscr{A}=\left\{T^{j} p(T): j \geqslant 0\right\} \subseteq \mathscr{U}$ for every polynomial $p$. We will show that $\mathscr{A} \cup\{T\}$ satisfies the hypotheses of Theorem 2.1 for a suitable decomposition of the space $\mathscr{H}$; hence $\mathscr{U}$ is strongly dense in $\mathscr{L}(\mathscr{H})$.

To this end, consider the subspace $\mathscr{M}=\mathscr{M}_{n}\left(\mathscr{M}_{n}=\mathrm{V}_{j \geqslant n+1} e_{j}\right)$. Clearly, $\mathscr{M}$ is invariant for $T$ and $T \mid \mathscr{M}$ is unitarily equivalent to the shift operator $S$. Thus, $T$ is represented by the operator matrix $\left(\begin{array}{ll}S & T_{12} \\ 0 & T_{22}\end{array}\right)$ on the space $\mathscr{H}=\mathscr{M} \oplus \mathscr{M}^{\perp}$, where the operators $T_{12}$ and $T_{22}$ are given by

$$
T_{12} e_{i}= \begin{cases}0 & \text { if } 1 \leqslant i \leqslant n-1 \\ e_{n+1} & \text { if } i=n\end{cases}
$$

and

$$
T_{22}=\left(t_{i j}\right)= \begin{cases}1+u_{i j} & \text { if } j=i-1 \\ u_{i j} & \text { otherwise }\end{cases}
$$


If $p(\lambda)=\sum_{j=0}^{n} a_{j} \lambda^{j}$ is the characteristic polynomial of $T_{22}$, then for each $j \geqslant 0$ we have

$$
T^{j} p(T)=\left[\begin{array}{cc}
S^{j} p(S) & S^{j} A \\
0 & 0
\end{array}\right], \quad A=\sum_{j=1}^{n} \sum_{i=0}^{j-1} a_{j} S^{j-i-1} T_{12} T_{22}^{i} .
$$

For each $i, 1 \leqslant i \leqslant n-1$, the vector $T_{22}^{i} e_{1}$ is orthogonal to the subspace $\mathscr{M}_{i+1}$; hence we have

$$
A e_{1}=\prod_{j=1}^{n}\left(1+u_{j+1, j}\right) e_{n+1}
$$

and thus

$$
\bigvee_{j \geqslant 0} S^{j} A e_{1}=\mathscr{M}
$$

The fact that $S$ has the TAP together with (1) and (2) implies that $\mathscr{A} \cup\{T\}$ satisfies the hypotheses of Theorem 2.1. Therefore, $\mathscr{U}$ is strongly dense in $\mathscr{L}(\mathscr{H})$ and hence $T$ has the TAP.

COROLlaRY 3.2. If $U$ is any finite upper triangular matrix, then the operator $U \oplus 0 \in \mathscr{L}(\mathscr{H})$ has the strong-TAP.

For the case $n=2$ we can strengthen this result:

THEOREM 3.3. If $U$ is any $2 \times 2$ matrix, then the operator $U \oplus 0 \in \mathscr{L}(\mathscr{H})$ has the strong-TAP.

Proof. Let $T=S+U \oplus 0$. If $u_{21} \neq-1$, then $T$ has the TAP by the previous theorem. Thus, we may assume that $u_{21}=-1$. Let $\mathscr{U}$ be a transitive operator algebra which contains the operator $T$. Consider the following triangular forms of $T$ :

$$
T=\left[\begin{array}{ll}
T_{11} & 0 \\
T_{21} & u_{11}
\end{array}\right] \in \mathscr{L}\left(\mathscr{M}_{1} \oplus \mathscr{M}_{1}^{\perp}\right)
$$

and

$$
T=\left[\begin{array}{ll}
S & T_{12} \\
0 & T_{22}
\end{array}\right] \in \mathscr{L}\left(\mathscr{M}_{2} \oplus \mathscr{M}_{2}^{\perp}\right) \text {. }
$$

The operator $T_{11}$ is unitarily equivalent to the operator $S+\left(u_{22}\right) \oplus 0$, hence $T_{11}$ has the TAP by Theorem 3.1. It now follows that

(3) $\bigvee\left(T_{11}-u_{11} I\right)^{*} \mathscr{M}_{1}+T_{21}^{*} e_{1}=\mathscr{M}_{1}$, unless $u_{12}=0$ and $u_{11}=u_{22} \neq 0$;

$$
\bigvee_{j \geqslant 0} S^{j} T_{12} e_{2}=\mathscr{M}_{2}, \quad \text { if } u_{12}=0 \text { and } u_{11}=u_{22} \neq 0
$$

If (3) holds, then $\left\{T^{*},\left(T-u_{11} I\right)^{*}\right\}$ satisfies the hypotheses of Theorem 2.1; hence $\mathscr{U}^{*}$ is strongly dense in $\mathscr{L}(\mathscr{H})$ (recall $\mathscr{U}^{*}$ is transitive if and only if $\mathscr{U}$ is transitive).

If (4) is satisfied, then condition ( $\dagger$ ) of Theorem 2.1 holds for $\left\{T^{j}\left(T-u_{11} I\right)\right.$ : $j \geqslant 0\}$; hence $\mathscr{U}$ is strongly dense in $\mathscr{L}(\mathscr{H})$. Thus, in any case the operator $T$ has the TAP. 
The following result somewhat improves Theorem 3.1 for the case $n=3$.

THEOREM 3.4. If $U$ is a $3 \times 3$ matrix such that none of $u_{21}, u_{32}$, and

$$
\gamma=u_{31}\left(1+u_{21}\right)^{-1}\left(1+u_{32}\right)^{-2}\left[\left(u_{11}-u_{22}\right)\left(1+u_{32}\right)-u_{31}\right]
$$

is equal to -1 , then the operator $U \oplus 0 \in \mathscr{L}(\mathscr{H})$ has the strong-TAP.

Proof. We will show that the operator $T=S+U \oplus 0$ has the TAP by producing a similarity between operators $T$ and $S+V \oplus 0$ for some almost upper triangular matrix $V$. To this end, let

$$
A=\left(\begin{array}{lll}
u_{11} & u_{12} & u_{13} \\
0 & u_{22} & u_{23} \\
0 & 0 & u_{33}
\end{array}\right), \quad B=U-A,
$$

and define the operator $X \in \mathscr{L}(\mathscr{H})$ by

$$
X=\left(I+S^{* 2}(B \oplus 0) S\right)\left(I+S^{*}(B \oplus 0)\right) .
$$

It is easy to verify that:

$$
X=\left(\begin{array}{cc}
\left(1+u_{21}\right)\left(1+u_{32}\right) & 0 \\
u_{31} & 1+u_{32}
\end{array}\right) \oplus I
$$

and

$$
X \text { is invertible and } X(S+(B \oplus 0)) X^{-1}=S \text {, }
$$

$$
\text { (7) } X A X^{-1}=\left(\begin{array}{ccc}
* & * & * \\
\gamma & * & * \\
0 & 0 & *
\end{array}\right) \text {. }
$$

Since $\gamma \neq-1, X A X^{-1}$ is an almost upper triangular matrix. Thus, the operator $S+X A X^{-1} \oplus 0$ has the TAP by Theorem 3.1. This fact together with (6) implies that the operator $S+U \oplus 0$ has the TAP.

We will now study the lower triangular cases.

LEMMA 3.5. Let $L=\left(l_{i j}\right)$ be strictly lower triangular and suppose that, for each $i$ $(1 \leqslant i \leqslant n), l_{i+1, i} \neq-1$. Then the perturbed shift operator $S+L \oplus 0$ is similar to the shift operator $S$.

Proof. We define the operator $X$ by

$$
X=\prod_{j=1}^{n-1}\left(I+S^{* n-j}(L \oplus 0) S^{n-j-1}\right)
$$

It is easy to see that $X$ is invertible and $X$ implements a similarity between operators $S+L \oplus 0$ and $S$.

COROLlaRY 3.6. Let $L$ be as in the statement of Lemma 3.5. Then the operator $L \oplus 0 \in \mathscr{L}(\mathscr{H})$ has the strong-TAP.

Let $J_{\lambda}$ denote the elementary Jordan matrix with the eigenvalue $\lambda$.

Lemma 3.7. If $M$ is any finite matrix such that $\sigma\left(M+J_{0}\right) \subseteq\{\lambda:|\lambda|>1\}$, then the operator $M \oplus 0 \in \mathscr{L}(\mathscr{H})$ has the strong-TAP. 
Proof. If $M$ is $n \times n$, then the operator $T=S+M \oplus 0$ has the triangular form $\left(\begin{array}{cc}S & A \\ 0 & M+J_{0}\end{array}\right)$ corresponding to the decomposition $\mathscr{H}=\mathscr{M}_{n} \oplus \mathscr{M}_{n}^{\perp}$. It follows from the hypothesis that

$$
\sigma(S) \cap \sigma\left(M+J_{0}\right)=\varnothing ;
$$

hence $T$ and $S \oplus\left(M+J_{0}\right)$ are similar [7, p. 9]. It is now evident from Theorem 2.1 that the operator $S \oplus\left(M+J_{0}\right)$ has the TAP; hence the operator $T$ has the TAP.

We now consider the analytic representation of the unilateral shift $S$. Recall the Hardy space $H^{2}$ as the class of all functions $f$ analytic in the disk $\mathbf{D}=\{z: z \in \mathbf{C}$, $|z|<1\}$ of the complex plane $\mathbf{C}$ such that

$$
f(z)=\sum_{j \geqslant 0} \hat{f}(j) z^{j}, \quad z \in \mathbf{D} ; \quad\|f\|^{2} \stackrel{\text { def }}{=} \cdot \sum_{j \geqslant 0}|\hat{f}(j)|^{2}<\infty .
$$

The shift operator $S \in \mathscr{L}\left(\mathscr{H}^{2}\right)$ is given by $(S f)(z)=z f(z)$.

Proposition 3.8. If $f: \mathbf{D} \rightarrow \mathbf{D}$ is analytic and if $f$ satisfies conditions $f(0) \neq 0$ and $f(f(0))=0$, then $f$ is a linear fractional transformation.

Proof. Define $g(z)=(\xi-z) /(1-\bar{\xi} z), \xi=f(0)$, and let $h=f \circ g^{-1}$. Observe that $h: \mathbf{D} \rightarrow \mathbf{D}$ is analytic and has two fixed points 0 and $\xi$. It follows from Schwartz's Lemma that $h$ is the identity; hence $f=g$.

We will use this proposition to prove Corollary 3.10.

For $f \in H^{2}, f \otimes 1 \in \mathscr{L}\left(H^{2}\right)$ is the rank one operator given by

$$
(f \otimes 1)(h)=h(0) f, \quad h \in H^{2} .
$$

THEOREM 3.9. If $f \in H^{2}$ satisfies any one of the three conditions

(i) $f(0)=0$,

(ii) $0<|f(0)|<1$ and $f(f(0)) \neq 0$, and

(iii) $|f(0)| \geqslant 1$,

then the operator $f \otimes 1$ has the strong-TAP.

Proof. Let $T=S+f \otimes 1$ and let $H_{j}=\mathrm{V}_{k \geqslant j} z^{k}, j=1,2$. Consider the following decompositions of $T$ :

$$
T=\left[\begin{array}{ll}
S & T_{12} \\
0 & \xi
\end{array}\right] \in \mathscr{L}\left(H_{1} \oplus H_{1}^{\perp}\right) \quad \text { and } \quad T=\left[\begin{array}{cc}
S & A \\
0 & B
\end{array}\right] \in \mathscr{L}\left(H_{2} \oplus H_{2}^{\perp}\right),
$$

where

$$
\begin{gathered}
T_{12}(1)=(1+\hat{f}(1)) z+\sum_{j=2}^{\infty} \hat{f}(j) z^{j}, \\
A\left(z^{k}\right)=(1-k)\left(\sum_{j=2}^{\infty} \hat{f}(j) z^{j}\right)+k z^{2}, \quad k=0,1,
\end{gathered}
$$

and

$$
B\left(z^{k}\right)=(1-k)[\xi+(1+\hat{f}(1)) z], \quad k=0,1 .
$$


It follows from properties of the shift and the definition of $H_{1}$ that

$$
z^{j}-\xi^{j-1} z \in(S-\xi) H_{1}, \quad j \geqslant 2 .
$$

We now have:

If $\xi=0$ and $\hat{f}(1) \neq-1$, then $\bigvee S H_{1}+T_{12} H_{1}^{\perp}=H_{1}$;

If $\xi=0$ and $\hat{f}(1)=-1$, then $B=0$ and $\bigvee S H_{2}+A H_{2}{ }^{\perp}=H_{2}$;

If $|\xi| \geqslant 1$, then $(1 / \xi)^{j-1} z^{j}-z \in(S-\xi) H_{1}, j \geqslant 2$ and hence

$$
\bigvee(S-\xi) H_{1}=H_{1} \text {. }
$$

Thus, if $f$ satisfies either (i) or (iii), then Theorem 2.1 applies and so $T$ has the TAP.

Now suppose $0<|\xi|<1$ and $f(\xi) \neq 0$. From (1) we have

$$
\sum_{j=2}^{\infty} \hat{f}(j) z^{j}-\left(\sum_{j=2}^{\infty} \hat{f}(j) \xi^{j-1}\right) z \in \bigvee(S-\xi) H_{1}
$$

hence

$$
\frac{f(\xi)}{\xi} z=\left(1+\sum_{j=1}^{\infty} \hat{f}(j) \xi^{j-1}\right) z \in \bigvee(S-\xi) H_{1}+T_{12} H_{1}^{\perp}
$$

It is now evident from (8) and (9) that condition ( $\dagger$ ) of Theorem 2.1 is satisfied for $T$; hence $T$ has the TAP.

Recall a function $\varphi \in H^{2}$ is called inner if $|\varphi(\lambda)|<1, \lambda \in \mathbf{D}$, and $\left|\varphi\left(e^{i \theta}\right)\right|=1$ for almost all $\theta$. This theorem together with the proposition implies:

COROLlaRY 3.10. If $\varphi \in H^{2}$ is any inner function which is not a single Blaschke factor $\left(\varphi \neq(\xi-z) /\left(1-\bar{\xi}_{z}\right), \xi \neq 0\right)$, then the operator $\varphi \otimes 1$ has the strong-TAP.

REMARK. This theorem was proved in [1] for the case $\xi=0$ and $\hat{f}(1) \neq-1$.

OPEN QUESTIONS. Our technique implies that every finite rank operator has the strong-TAP, if the answer to the following question is affirmative:

Question 1. If condition ( $\dagger$ ) of Theorem 2.1 is replaced by the weaker condition

$$
\bigvee\left(A \mathscr{H}+B \mathscr{K}:\left[\begin{array}{cc}
A & B \\
0 & 0
\end{array}\right] \in \mathscr{A}\right)=\mathscr{H}-\mathscr{M},
$$

where $\mathscr{M}$ is a finite-dimensional subspace of $\mathscr{H}$, is the assertion of the theorem still true?

Question 2. If $\varphi(\varphi(0) \neq 0)$ is a linear fractional transformation of the open unit disk, must $\varphi \otimes 1$ have the strong-TAP?

A weakly closed unital operator algebra $\mathscr{U}$ is reductive if $\mathscr{L}$ at $\mathscr{U}=\mathscr{L}$ at $\mathscr{U}^{*}$. Von Neumann algebras are clearly reductive; the reductive algebra problem is the question: Must every reductive operator algebra be a von Neumann algebra? For the discussion of partial results consult [7, Chapter 9]. Almost all the partial solutions of the transitive algebra problem have been extended to the reductive algebra problem. In [2], we presented a result of this kind. Therefore, one is led to ask:

Question 3. If a reductive operator algebra $\mathscr{U}$ contains an operator with the TAP, must $\mathscr{U}$ be a von Neumann algebra? 
Acknowledgment. The author wishes to thank Professors Joel Anderson and Allen Shields for many useful conversations.

\section{REFERENCES}

1. M. A. Ansari, Transitive algebra containing triangular operator matrices, J. Operator Theory 14 (1985), 173-180.

2. __ Reductive algebras containing a direct sum of the unilateral shift and a certain other operator are selfadjoint, Proc. Amer. Math. Soc. 93 (1985), 284-286.

3. The weak-star generators of $e_{n}(z)=z^{n}(|z|<1)$ (to appear).

4. W. B. Arveson, $A$ density theorem for operator algebras, Duke Math. J. 34 (1967), 635-647.

5. K. J. Harrison, W. E. Longstaff, and P. Rosenthal, Some tractable non-selfadjoint operator algebras, J. London Math. Soc. (2) 26 (1982), 325-330.

6. B. Moore III and E. Nordgren, On transitive algebras containing $C_{0}$-operators, Indiana Univ. Math. J. 24 (1975), 777-789.

7. H. Radjavi and P. Rosenthal, Invariant subspaces, Springer-Verlag, Berlin and New York, 1972.

Department of Mathematics, Pennsylvania State University, University Park, Pennsylvania 16802

Current address: Penn State Berks Campus, Department of Mathematics, P. O. Box 7009, Reading, Pennsylvania 19610-6009 